\section{Eine neue Allianz für hautkranke Kinder}

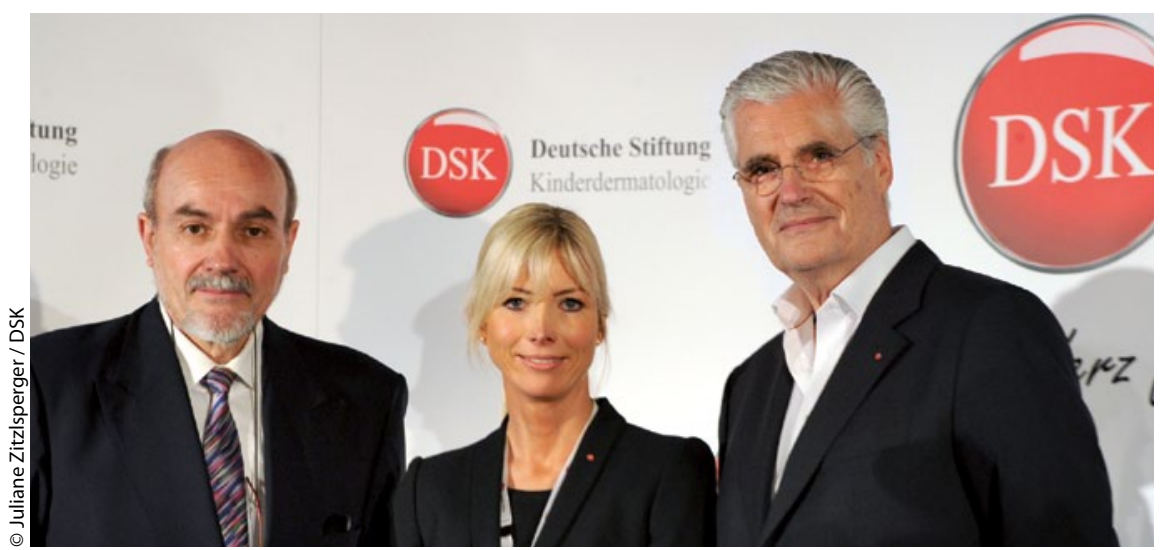

Stiftungsgründerin Dr. Nicole Brandl, eingerahmt von DSK-Schirmherr Prof. Dr. Dr. Johannes Ring (links) und DSK-Botschafter Sky du Mont

A n der Schnittstelle zwischen Pädiatrie und Dermatologie fallen hautkranke Kinder leider allzu oft durch das Raster des öffentlichen Gesundheitswesens. Diesen Missstand zu ändern, ist das erklärte Ziel der neu gegründeten Deutschen Stiftung Kinderdermatologie (DSK). Stifterin ist die Münchner Hautärztin Dr. Nicole Brandl, die ihr Projekt im März in einer Pressekonferenz erstmals vorstellte: „Kinder benötigen spezielle Therapien und Schulungen, mehr Forschung und sozialverträgliche Versorgungsstrukturen“, so ihr Credo. Mit an Bord sind prominente Vertreter aus Pädiatrie wie Dermatologie, was angesichts des nicht immer unproblematischen Verhältnisses beider Fachrichtungen bemerkenswert ist.

Prof. Dr. Dr. Johannes Ring von der Hautklinik der TU München stellte als Schirmherr der Stiftung deren Förderprogramm vor: „Das Programm zielt darauf $a b$, die in weiten Teilen Deutschlands ungenügende dermatologische Versorgungslage von hautkranken Kindern und Jugendlichen sowie deren Angehöriger nachhaltig zu verbessern. "Die Stiftungsarbeit beinhaltet dabei die Etablierung von Schulungsprogrammen sowie die Förderung von Lehr- und Forschungsvorhaben - etwa zur Entwicklung spezifischer Behandlungsformen, zur Validierung von Off-Label-Therapien oder zur Sammlung epidemiologischer Daten. Der Aufbau von Zentren für Kinder- und Jugenddermatologie ist das Fernziel.
Um diese großen Pläne realisieren $\mathrm{zu}$ können, ist jede Hilfe willkommen. Getreu der Philosophie „Du bist die Stiftung“ ist dabei ehrenamtlich gespendete Zeit mindestens genauso wertvoll wie finanzielle Zuwendungen. Prominente und Künstler können zudem die Öffentlichkeitsarbeit unterstützen, als ersten Botschafter konnte die DSK den Schauspieler Sky du Mont gewinnen.

es
Am Samstag, den 15. Oktober 2011 lädt das Netzwerk interdisziplinäre pädiatrische Dermatologie (NipD e.V.) zur Jahrestagung nach München ein. Die Veranstaltung hat zum Ziel, die Zusammenar\section{NipD e.V.}

\section{Programm:}

9:15 Begrüßung Prof. Dr. med. Burdach / Prof. Dr. Cremer

9:30 Häufige Fragen in der Dermatotherapie des Kindesalters PD Dr. Schnopp

\title{
Phyto-Forum: \\ Experten beraten online
}

"Gegen alles ist ein Kraut gewachsen", sagt der Volksmund. Das macht deutlich: Pflanzen und Kräuter spielen in der Heilkunst seit Jahrhunderten eine wichtige Rolle. Und auch heute haben pflanzliche Arzneien einen festen Platz in der Pharmakotherapie. Mit dem OnlineForum zur Phytotherapie bietet die "Ärzte Zeitung" einen neuen Service. Unter www.aerztezeitung.de können sich Angehörige medizinischer Fachkreise kostenlos mit Fragen an Experten wenden. Was kann Phytotherapie leisten? Worauf beruht die pharmakologische Wirkung pflanzlicher Präparat? Auf welche Wechselwirkungen ist zu achten? Wo haben sich pflanzliche Arzneimittel besonders bewährt? Zu Phytotherapeutika gibt es immer wieder Fragen - von den Ärzten selbst oder von den Patienten an ihre Ärzte. Diese werden nun im Phyto-Forum von fünf Mitgliedern der Gesellschaft für Phytotherapie beantwortet.

\section{Fortbildungsveranstaltung des NipD}

beit von Pädiatern und Dermatologen zu fördern. Tagungsort ist die Klinik und Poliklinik für Kinder- und Jugendmedizin, Klinikum Schwabing, Kölner Platz 1, 80804 München.
10:10 Vaskuläre Malformationen mit Abgrenzung zu Hämangiomen Prof. Dr. Grantzow

10:40 Propranol - systemisch und lokal in der Therapie von „Problem-Hämangiomen" Dr. Schneider

11:20 Fallvorstellung: Epidermolysis bullosa dsystrophica Mitarbeiter der Klinik Schwabing
13:00 Mittagspause

14:00 Melanozytäre

Naevi

Prof. Dr. Cremer

14:40 Frühe Resektion kongenitaler melanozytärer Riesennävi mittels Powerdehnung der Haut

Prof. Dr. Breuninger

14:45 Akne im Kindesalter

PD. Dr. Jansen 